\title{
Erratum: The Effects of Perception of Efficacy and Diagram Construction Skills on Students' Spontaneous Use of Diagrams When Solving Math Word Problems ${ }^{\star}$
}

\author{
Yuri Uesaka ${ }^{1}$, Emmanuel Manalo $^{2}$, and Shin'ichi Ichikawa ${ }^{3}$ \\ 1 Japan Society for the Promotion of Science, Tokyo Institute of Technology \\ 2 Waseda University \\ 3 The University of Tokyo \\ y.uesaka@nm.hum.titech.ac.jp, emmanuel.manalo@gmail.com \\ ichikawa@p.u-tokyo.ac.jp
}

\begin{abstract}
A.K. Goel, M. Jamnik, and N.H. Narayanan (Eds.): Diagrams 2010, LNAI 6170, pp. 197-211, 2010.
(C) Springer-Verlag Berlin Heidelberg 2010
\end{abstract}

DOI: 10.1007/978-3-642-14600-8_51

Table 2 and Table 3 of the paper starting on page 197 of this volume are incorrect. Here are the correct versions:

Table 2. Ratios of Participants Referring to the Efficacy of Diagram Use in the Free Comments Section of the Post-instruction Survey

\begin{tabular}{llll}
\hline & With PD & Without PD & Totals \\
\hline With VE & $0.46(11 / 24)$ & $0.42(8 / 19)$ & $0.44(19 / 43)$ \\
\hline Without VE & $0.10(2 / 21)$ & $0.18(4 / 22)$ & $0.14(6 / 43)$ \\
\hline Totals & $0.29(13 / 45)$ & $0.29(12 / 41)$ & $0.29(25 / 86)$ \\
\hline
\end{tabular}

Note. In parentheses are the number of participants who referred to the efficacy of diagram use, and the total number of participants, in each condition.

Table 3. The Total Number of Problems for which Participants Produced High Quality Diagrams When Asked to Use Diagrams in the Post-instruction Assessment

\begin{tabular}{llll}
\hline & With PD & Without PD & Totals \\
\hline With VE & $2.46(0.59)$ & $1.95(0.91)$ & $2.23(0.78)$ \\
\hline Without VE & $2.19(0.75)$ & $1.77(1.06)$ & $1.98(0.94)$ \\
\hline Totals & $2.46(0.59)$ & $1.95(0.91)$ & $2.23(0.78)$ \\
\hline
\end{tabular}

In the original version, the chi-square symbol $(\chi)$ had inadvertently become the division sign $(\div)$ on pages 205 (at the bottom), 206 and 207 (at the top).

\footnotetext{
^ The original online version for this chapter can be found at: http://dx.doi.org/10.1007/978-3-642-14600-8_19
} 